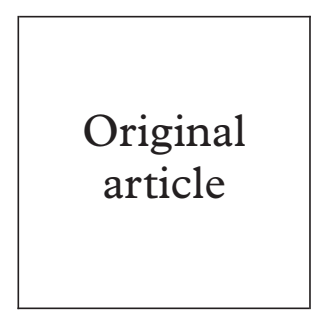

National Center for Infectious Diseases, Centers for Disease Control and Prevention, Atlanta, GA, USA

C I Bandea

K Kubota

T M Brown

V Bhullar

C M Black

The HIV/AIDS

Collaboration,

Nonthaburi, Thailand

P H Kilmarx

National Center for HIV, STD, and TB

Prevention, Centers for Disease Control and Prevention,

Atlanta, GA, USA

P H Kilmarx

Chiang Rai Hospital, Chiang Rai, Thailand $S$ Yanpaisarn

Siriraj Hospital, Bangkok, Thailand

P Chaisilwattana

Rajavithi Hospital, Bangkok, Thailand W Siriwasin

Correspondence to: Carolyn M Black, PhD, Mai stop C-17, Centers for Disease Control and Prevention, 1600 Clifton Road, Atlanta, GA 30333, USA

cblack@cdc.gov

Accepted for publication 22 May 2001

\title{
Typing of Chlamydia trachomatis strains from urine samples by amplification and sequencing the major outer membrane protein gene (omp1)
}

\author{
C I Bandea, K Kubota, T M Brown, P H Kilmarx, V Bhullar, S Yanpaisarn, \\ P Chaisilwattana, W Siriwasin, C M Black
}

Objectives: To develop a novel protocol for the extraction, amplification, and sequencing of Chlamydia trachomatis MOMP gene (omp1) from urine, a non-invasive source, and apply it to an epidemiological study on the distribution of $C$ trachomatis strains in a population of pregnant women in Thailand.

Methods: The $C$ trachomatis DNA was extracted from culture stocks and urine using a slightly modified commercially available kit, the High Pure PCR Template Preparation Kit (Roche Molecular Biochemicals, IN, USA). The PCR and sequencing primers used for the amplification and sequencing of the omp1 were designed based on the nucleotide sequence of multiple $C$ trachomatis strains found in GenBank. The protocol for the extraction, amplification, and sequencing was tested on laboratory culture stocks of reference strains of all $C$ trachomatis serovars and on urine samples collected in a cross sectional study designed to assess the prevalence of $C$ trachomatis infections in the cities of Bangkok and Chiang Rai, Thailand.

Results: The omp1 gene was successfully amplified and sequenced from 18 laboratory $C$ trachomatis reference strains and from $45 C$ trachomatis positive urine clinical samples collected from asymptomatic pregnant women. Among clinical samples, we found nine different $C$ trachomatis genotypes: $\mathrm{F}(11,25 \%), \mathrm{D}(10,22.6 \%), \mathrm{H}(5,11.7 \%), \mathrm{K}(5,11.7 \%), \mathrm{E}(4,9.3 \%)$, Ia $(3,7 \%), \mathrm{B}(3,7 \%), \mathrm{Ja}(2,4.5 \%)$, and $\mathrm{G}(1,2.3 \%)$. One specimen generated an omp 1 DNA sequence pattern indicating the presence of a mixed infection with at least two different serovars.

Conclusions: Urine is a convenient and reliable source for genotyping $C$ trachomatis strains. A clear advantage of urine over traditional samples, such as cervical swabs, is that urine is a noninvasive source which makes collection easier and thus facilitates the enrolment of patients in clinical and epidemiological studies. In addition to typing, urine is increasingly used for diagnosis of $C$ trachomatis infection by several commercially available nucleic acid amplification assays which represents a distinct advantage for collecting, transport, storage, and laboratory handling of samples. (Sex Transm Inf 2001;77:419-422)

Keywords: genotype; Chlamydia trachomatis; urine

\section{Introduction}

Chlamydia trachomatis infections are the leading cause of bacterial sexually transmitted diseases and the main cause of preventable blindness in the world. ${ }^{1} C$ trachomatis strains have been conventionally classified into serotypes or serovars by immunotyping using polyclonal or monoclonal antibodies specific for the major outer membrane protein (MOMP). Fifteen prototypic serovars labelled $\mathrm{A}$ to $\mathrm{K}$ and $\mathrm{L}_{1}, \mathrm{~L}_{2}$, and $\mathrm{L}_{3}$ were initially recognised by polyclonal antibodies, and additional immunovariants ( $\mathrm{Ba}, \mathrm{Da}, \mathrm{Ia}$, etc), which in some publications are referred to as distinct serovars, have been identified by monoclonal antibodies. ${ }^{2-4}$ Serovars $\mathrm{A}, \mathrm{B}$, and $\mathrm{C}$ have been found usually associated with trachoma, $\mathrm{D}$ to $\mathrm{K}$ with urogenital infections, and $\mathrm{L}_{1}, \mathrm{~L}_{2}$, and $\mathrm{L}_{3}$ with lymphogranuloma venereum (LGV), a systemic disease. The urogenital serovars are found all regions of the world, whereas the LGV and trachoma serovars are found primarily in tropical and subtropical regions. ${ }^{1}$

Typing of $C$ trachomatis strains is an important goal in field of epidemiology as well as clinical and basic research on $C$ trachomatis infections. The temporal and geographical distribution of $C$ trachomatis strains throughout the world has significant implications for our understanding of the epidemiology of this infectious agent and for vaccine development. ${ }^{5-8}$ Differential pathogenicity and transmission rates of $C$ trachomatis strains are also important topics in both clinical and basic research. ${ }^{9-13}$ The traditional immunotyping methods for the identification of $C$ trachomatis strains require collection of invasive samples, such as cervical swabs, and culture isolation and growth of the organisms. The immunotyping methods are currently being replaced by genotyping methods using restriction fragment length polymorphism (RFLP) or DNA sequence analyses of the major outer membrane protein (MOMP) gene (omp1). ${ }^{10-23}$ Compared with immunotyping, the genotyping methods, particularly omp 1 sequencing, are more sensitive and precise in revealing $C$ trachomatis variants within a serovar as well as potential recombinants among serovars. ${ }^{24}$ An additional advantage of the genotyping methods is that they have the potential to be used with non-invasive samples such as urine, which is also the source of choice for current commercially available nucleic acid amplification diagnostic assays (NAA). In this paper we report the development of a protocol for the extraction, amplification 


\section{Key messages}

- The objective of the study was to develop a novel protocol for the extraction, amplification, and sequencing of $C$ trachomatis MOMP gene (omp1) from the urine, a non-invasive source, and apply it to an epidemiological study regarding the distribution of $C$ trachomatis strains in a population of pregnant women in Thailand.

- The omp1 gene was successfully amplified and sequenced from 18 laboratory $C$ trachomatis reference strains and from $45 C$ trachomatis positive urine clinical samples collected from asymptomatic pregnant women.

- A clear advantage of urine over traditional samples, such as cervical swabs, is that urine is a non-invasive source which makes collection easier and thus facilitates the enrolment of patients in clinical and epidemiological studies. In addition to typing, urine is increasingly used for diagnosis of $C$ trachomatis infection by several commercially available nucleic and amplification assays which represents a distinct advantage for collecting, transport, storage, and laboratory handling of samples.

and sequencing of $C$ trachomatis MOMP gene from urine samples collected from a population of pregnant women in Thailand.

\section{Materials and methods}

First catch urine $(30 \mathrm{ml})$ was collected in 1996 from pregnant women $(n=1021)$ enrolled in a cross sectional study designed to assess the prevalence of $C$ trachomatis, Neisseria gonorrhoeae, Treponema pallidum, and HIV infections in the cities of Bangkok (Siriraj and Rajavithi Hospitals) and Chiang Rai (Chiang Rai Hospital), Thailand. The demographic and clinical characteristics of the study population have been previously described. ${ }^{25}$ The protocol for this study was reviewed and approved by institutional review boards at the Centers for Disease Control and Prevention (CDC) and the hospitals in Thailand. The urine samples were shipped on dry ice to the CDC, Atlanta, where they were screened for $C$ trachomatis and $N$ gonorrhoeae by using a commercial polymerase chain reaction (PCR) assay (Amplicor CT/NG, Roche Molecular Systems, IN, USA) according to the manufacturer's instructions.

The Amplicor positive urine samples were stored at $-20^{\circ} \mathrm{C}$. The nested PCR amplification of the omp1 gene was performed as follows: $200 \mu \mathrm{l}$ of urine was centrifuged at 1000 $g$ for 15 minutes in a microcentrifuge; the supernatant was discarded and the pellet was resuspended in $200 \mu \mathrm{l}$ of buffer ( $10 \mathrm{mM}$ TRIS$\mathrm{HCl}, \mathrm{pH} 7.5,0,05 \%$ Triton $\mathrm{X}), 400 \mu \mathrm{l}$ of binding buffer, and $40 \mu \mathrm{l}$ of proteinase $\mathrm{K}$ from the High Pure PCR Template Preparation Kit (Roche Molecular Biochemicals, IN, USA) and incubated at $72^{\circ} \mathrm{C}$ for 60 minutes. The

Table 1 PCR and sequencing primers used for amplifying and sequencing the omp1 gene of $C$ trachomatis strains

\begin{tabular}{ll}
\hline First PCR primers: & \\
CT90UF & 5'-GGACATCTTGTCTGGCTTTAACT-3' \\
CT220DR & 5'-GCGCTCAAGTAGACCGATATAGTA-3' \\
Secondary PCR primers: & 5'-GTCCCGCCAGAAAAAGATAG-3' \\
CT60UF & 5'-CCAGAAACACGGATAGTGTTATTA-3' \\
CT80DR & 5'-ATAGCGAGCACAAAGAGAGC-3' \\
Sequencing primers: & 5'-ACCACTTGGTGTGACGCTATCCAG-3' \\
CT40F & 5'-TGGGATCGTTTTGATGTATT-3' \\
CT160F & 5'-ACGTTAGGAGCTTCTTTCCATA-3' \\
CT419F & 5'-TCCTTACATTGGAGTTAAATGGTC-3' \\
CT662F & \\
CT902F &
\end{tabular}

DNA was further purified using the High Pure PCR Template Preparation Kit and suspended in $200 \mu$ l elution buffer according to the manufacturer's instructions. Ten $\mu$ of the prepared DNA template, which represented the equivalent of $10 \mu \mathrm{l}$ of urine, was used in the $100 \mu \mathrm{l}$ primary PCR reaction (High Fidelity Expand Kit, Roche) using first PCR primers CT90UF and CT220DR located upstream and downstream, respectively, of the omp1 coding region (table 1). The thermocycler profile was: $94^{\circ} \mathrm{C}$ for 30 seconds, $52^{\circ} \mathrm{C}$ for 1 minute, and $72^{\circ} \mathrm{C}$ for 1 minute for 40 cycles. Two $\mu$ l of the primary PCR were used for secondary PCR which was prepared and run using the same reagents and conditions as the primary PCR, except for primers, which were CT60UF and CT80DR. The laboratory culture stocks of 18 reference $C$ trachomatis strains (A to $\mathrm{K}, \mathrm{L}_{1}-\mathrm{L}_{3}, \mathrm{Da}$, Ja, and Ia) were prepared and amplified using the same protocol.

The amplified DNA was purified using the Qiagen Qiaquick PCR Purification Kit (Qiagen, Santa Clarita, CA, USA) as instructed by the manufacturer. The omp1 was sequenced using the Big Dye Sequencing Terminator Kit and ABI Prism 377 automated sequencing system (PE-Applied Biosystems, Foster City, CA, USA) and five sequencing primers: CT40F, CT160F, CT419F, CT662F, and CT902F (table 1).

The omp1 sequences were edited, aligned, and analysed using the software Wisconsin Package Version 10.0 (GCG, Madison, WI, USA).

\section{Results}

The entire coding region of omp 1 from 18 laboratory reference strains representing all of the serovars and some immunovariants of $C$ trachomatis was successfully amplified and sequenced using the protocol and the primers described above in the methods section. One round of PCR was sufficient to generate enough DNA from culture stocks to perform multiple rounds of sequencing (data not shown).

All $45 C$ trachomatis positive urine samples that were available for this genotyping study were also successfully amplified. However, for consistent results and to obtain enough amplified DNA to perform multiple rounds of sequencing reactions, a nested PCR procedure was necessary. Presumably, the need for a nested PCR procedure was the result of a low number of $C$ trachomatis organisms in some urine samples or, potentially, because of PCR inhibitors present in urine.

Except for one sample that generated a DNA sequence pattern indicating a mixed infection with at least two different strains, all of the remaining 44 sequences showed the presence of a single or a predominant strain. The sequencing procedure used in this study can not detect mixed infections when the molar ratio of the amplified mixed strains is higher than five to one (data not shown). Among the 44 Thai sequences analysed by sequence comparison and phylogenetic analysis, nine different serovars were identified: F (11, 25\%), D 
$(10,22.6 \%), \mathrm{H}(5,11.7 \%), \mathrm{K}(5,11.7 \%), \mathrm{E}(4$, $9.3 \%), \mathrm{Ia}(3,7 \%), \mathrm{B}(3,7 \%), \mathrm{Ja}(2,4.5 \%)$, and $\mathrm{G}(1,2.3 \%)$.

Discussion

We have developed a protocol for genotyping $C$ trachomatis strains from urine samples and applied it to a preliminary epidemiological study of the distribution of $C$ trachomatis serovars among pregnant women in Thailand. Firstly, we used this protocol to amplify and sequence laboratory strains representing all $C$ trachomatis serovars. Then we successfully extracted, amplified, and sequenced the omp1 gene from 45 urine samples collected from pregnant women in Thailand. Overall, the distribution of $C$ trachomatis serovars among pregnant women in Thailand appears to be similar to that observed in non-pregnant women living in Western countries, in which $\mathrm{E}$, $\mathrm{D}$, and $\mathrm{F}$ are the predominant serovars. In Thailand, we found that $\mathrm{F}(25 \%)$ and $\mathrm{D}$ $(22.6 \%)$ were the most common serovars, followed by more or less equal distributions of serovars $\mathrm{H}(11.7 \%), \mathrm{K}(11.7 \%)$, and $\mathrm{E}(9.3 \%)$. A similar distribution of $C$ trachomatis serovars among asymptomatic pregnant women in the same region of the world was recently reported from Japan. ${ }^{26}$ In this study the $C$ trachomatis serovars were identified from cervical samples by RFLP and partial $o m p 1$ sequencing.

The use of urine samples for typing of $C$ trachomatis strains presents distinct advantages compared with other samples, such as cervical swabs, particularly when conducting large epidemiological investigations. Urine is a noninvasive source which makes collection easier and thus facilitates the enrolment of patients. It also enables easier access to asymptomatic people and those who are not seeking clinical care. In this regard it should be particularly useful for certain epidemiological applications, such as partner transmission studies, in which the convenience of sample collection is key to successful enrolment of participants. Urine samples are also relatively stable at refrigerated temperatures and if necessary can be collected in relatively large volumes that permit repeat testing when required.

Another significant advantage in using urine for typing $C$ trachomatis strains is that it can also be used for the diagnosis of $C$ trachomatis infection by several commercially available NAA. ${ }^{27}$ Collecting only one sample for both diagnostic testing and for genotyping helps with enrolment of patients and with collecting, transport, storage, and laboratory handling of samples. Also, the NAA diagnostic tests, which are semiautomated and highly sensitive, can be used as a screening tool for identifying $C$ trachomatis positive samples for typing.

Interest in genotyping $C$ trachomatis directly from urine has been recently highlighted by Morre et al who outlined the advantages of using this source for epidemiological studies and successfully used urine in their PCR based RFLP genotyping protocol. ${ }^{28}$ They showed that, as expected, the same $C$ trachomatis strains are present in urine and cervical samples collected from the same patient. Their success rate of amplifying a portion of the omp 1 gene from $C$ trachomatis positive urine samples from females and males was $94 \%(n=81)$. In our study, by using samples from pregnant women we were able to amplify the entire omp 1 gene from all 45 urine samples available for this study.

In summary, urine represents a convenient and reliable source for genotyping $C$ trachomatis strains. As a non-invasive source, it can be easily collected from symptomatic and asymptomatic patients enrolled in epidemiological and clinical studies designed to address $C$ trachomatis pathogenicity, transmission, and vaccine development.

We thank Ms Azita Eslamiyan, Ms Nancy Young, Ms Supaporn Korattana, Mr Jirasak Laosakkitiboran, and $\mathrm{Dr}$ Prayoon Korattana, $\mathrm{Mr}$ Jirasak Laosakkitiboran, and Dr Prayoon
Yuentrakul, for their assistance in performing this research; Dr Yuentrakul, for their assistance in performing this research; Dr
Nathan Shaffer for his assistance and critical review of the Nathan Shaffer for his assistance and critical review of the
manuscript; and Dr David Martin for critical review of the manuscript;

Contributors: CIB, PHK, and CMB were responsible for study design and supervision, data analysis, and preparation of the manuscript; SY, PC, and WS were responsible for subjects' recruitment and preparation of manuscript; CIB, KK, TMB, and VB were the laboratory based scientists who, in addition to data analysis and manuscript preparation, were responsible for laboratory testing.

1 Schachter J. Infection and disease epidemiology. In: Stephens RS, ed. Chlamydia: intracellular biology, pathogenesis, and immunity. Washington DC: ASM, 1999.

2 Wang SP, Kuo CC, Barnes RC, et al. Immunotyping of Chlamydia trachomatis with monoclonal antibodies. $f$ Infect Dis 1985;152:791-800.

3 Wang SP, Grayston JT. Three new serovars of Chlamydia trachomatis: Da, Ia, and L2a. F Infect Dis 1991;163:403-5. trachomatis: Da, Ia, and L2a. F Infect Dis 1991;163:403-5.
4 Ossewaarde JM, Rieffe M, de Vries A, et al. Comparison of two panels of monoclonal antibodies for determination of Chlamydia trachomatis serovars. F Clin Microbiol 1994;32: 2968-74.

5 Caldwell HD, Schachter J. Antigenic analysis of the major outer membrane protein of Chlamydia spp. Infect Immun 1982;35:1024-31.

6 Brunham RC, Plummer FA, Stephens RS. Bacterial antigenic variation, host immune response, and pathogenhost coevolution. Infect Immun 1993;61:2273-6.

7 Brunham RC. Human immunity to Chlamydiae. In: Stephens RS, ed. Chlamydia: intracellular biology, pathogenesis, and immunity. Washinton DC: ASM, 1999.

8 Grayston JT, Wang S-P. The potential for vaccine against infection of the genital tract with Chlamydia trachomatis. Sex Transm Dis 1978;5:73-7.

9 Workowski KA, Stevens CE, Suchland RJ, et al. Clinical manifestations of genital infection due to Chlamydia manifestations of genital infection due to Chlamydia Infect Dis 1994;19:756-60.

10 Dean D, Oudens E, Bolan G, et al. Major outer membrane protein variants of Chlamydia trachomatis are associated with severe upper genital tract infections and histopathology in San Francisco. F Infect Dis 1995;172:1013-22.

1 Van Duynhoven YT, Ossewaarde JM, Derksen-Nawrocki $\mathrm{RP}$, et al. Chlamydia trachomatis genotypes: correlation with clinical manifestations of infection and patients' characteristics. Clin Infect Dis 1998;26:314-22.

12 Stothard DR, Boguslawski G, Jones RB. Phylogenetic analysis of the Chlamydia trachomatis major outer membrane protein and examination of potential pathogenic determinants. Infect Immun 1998;66:3618-25.

13 Dean D, Millman K. Molecular and mutation trends analyses of omp1 alleles for serovar $\mathrm{E}$ of Chlamydia trachomatis: Implications for the immunopathogenesis of disease. $\mathcal{F}$ Clin Invest 1997;99:475-83.

14 Hayes LJ, Pecharatana S, Bailey RL, et al. Extent and kinetics of genetic change in the omp 1 gene of Chlamydia ics of genetic change in the ompl gene of Chlamydia Dis 1995;172:268-72.

15 Dean D, Patton M, Stephens RS. Direct sequence evaluation of the major outer membrane protein gene variant regions of Chlamydia trachomatis subtypes D', I', and L2'. Infect Immun 1991;59:1579-82.

16 Stephens RS, Sanchez-Pescador R, Wagar EA, et al. Diversity of Chlamydia trachomatis major outer membrane protein genes. $\mathcal{F}$ Bacteriol 1987;169:3879-85.

17 Yuan Y, Zhang YX, Watkins NG, et al. Nucleotide and deduced amino acid sequences for the four variable domains of the major outer membrane proteins of the 15 Chlamydia trachomatis serovars. Infect Immun 1989;57: 1040-9.

18 Yang CL, Maclean I, Brunham RC. DNA sequence polymorphism of the Chlamydia trachomatis omp1 gene. $\mathcal{F}$ Infect Dis 1993;168:1225-30.

19 Frost EH, Deslandes S, Veilleux S, et al. Typing Chlamydia trachomatis by detection of restriction fragment length 
polymorphism in the gene encoding the major outer membrane protein. F Infect Dis 1991;163:1103-7. Chlamydia trachomatis by restriction endonuclease analysis of the amplified major outer membrane protein gene. $f$ Clin Microbiol 1991;29:1132-6.

21 Gaydos CA, Bobo L, Welsh L, et al. Gene typing of Chlamydia trachomatis by polymerase chain reaction and restriction endonuclease digestion. Sex Transm Dis 1992; 19:303-8.

22 Brunham RC, Kimani J, Bwayo J, et al. The epidemiology of Chlamydia trachomatis within a sexually transmitted diseases core group. F Infect Dis 1996;173:950-6.

23 Sayada C, Denamur E, Orfila J, et al. Rapid genotyping of the Chlamydia trachomatis major outer membrane protein by the polymerase chain reaction. FEMS Microbiol Lett 1991;67:73-8.

24 Hayes LJ, Yearsley P, Treharne JD, et al. Evidence for naturally occurring recombination in the gene encoding the major outer membrane protein of lymphogranuloma venereum isolates of Chlamydia trachomatis. Infect Immun 1994;62:5659-63

25 Kilmarx PH, Black CM, Limpakarnjanarat K, et al. Rapid assessment of sexually transmitted diseases in a sentinel population in Thailand: prevalence of chlamydial infection, gonorrhoea, and syphilis among pregnant women-1996. Sex Transm Inf 1998;74:189-93.

26 Ikehata M, Numazaki K, Chiba S. Analysis of Chlamydia trachomatis serovars in endocervical specimens derived from pregnant Japanese women. FEMS Immunol Med Microbiol 2000;27:35-41.

27 Black CM. Current methods of laboratory diagnosis of Chlamydia trachomatis infections. Clin Microbiol Rev 1997; 10:160-84.

28 Morre SA, Moes R, Van Valkengoed I, et al. Genotyping of Chlamydia trachomatis in urine specimens will facilitate large epidemiological studies. F Clin Microbiol 1998;36: 3077-8.

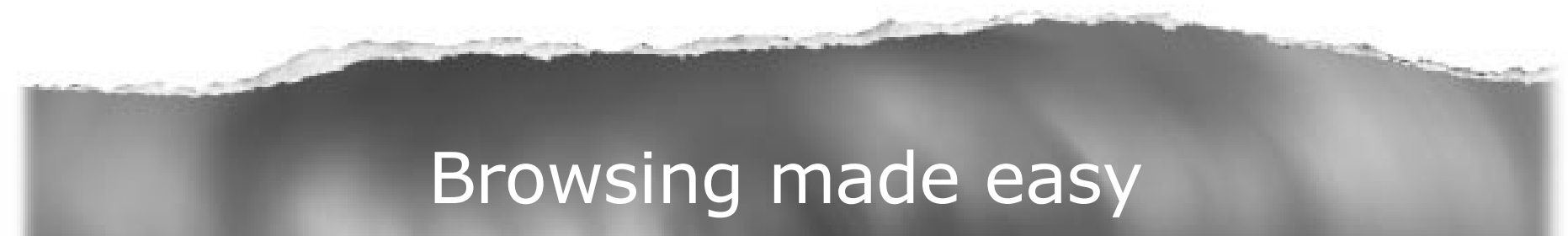

\section{Collections}

With a single click Collections allows you to find all articles that have been published in your chosen subject. Select from over 200 clinical and non-clinical topic collections and/or cross search other specialist journals, the BMJ and Cochrane Reviews

www.sextransinf.com 